resistance coupling of cells. Uncoupling of cells in development may prove to be an exciting clue to the mechanism of control. Aspects of differentiation relating to cell or tissue position are examined in a splendidly iconoclastical spirit (McMahon and West) in both regulating and non-regulating systems. Most of the important research dealing with embryonic induction has been compiled by Saxén et al. Cell surface specificity in cell systems in vitro seems to be present (Maslow), but its relevance to actual developmental events can always be questioned.

Five of the reviews deal with the development of the mammalian conceptus and placenta. They might have been more usefully placed in one of the later volumes. Gwatkin restricts his consideration of the organisation of the cell surface of gametes to the mouse. This seems a pity since much of the interesting work in this field lies in invertebrate studies. Sherman and Wudl make provocative comments

\section{Solar planets}

The Solar Planets. By V. A. Firsoff. Pp. 184. (David and Charles: Newton Abbot, London and Vancouver; Crane, Russak : New York, 1977.) $£ 5.50$.

CRUDEIY one can divide science books into four categories, elementary and advanced, good and bad. Firsoff's book is elementary and bad.

There is nothing wrong of course with a book being elementary. This one is written for the amateur astronomer and purports to be a study of the Solar System written in the light of the wealth of new knowledge gleaned from the unmanned space probes which have recently visited Mercury, Venus, Moon, Mars, Jupiter and Saturn. True, the book contains plenty of details about these missions, seventeen black and white plates and no formulae. And obviously the book is not all wrong, in fact a good $95 \%$ is right, but the unsuspecting reader has no way of picking out right from wrong, fact from the authors' flight of fantasy, the solid ground from the castles in Spain.

We are told: "it seems that the Moon began its existence as an independent planet and was later captured by the Earth", "a limestone meteorite fell in 1926 at Bleckenstad and is said to have contained fossils"; "Pluto may possibly belong to an older solar system or else be an immigrant from interstellar space"; "degeneracy is a condition derived from equations based on various simplifications which are always suspect". "I am not too sure that even in the Solar System ours is the only technical civilisation", "geography is fast becoming only a division about the putative role of the blastocyst in implantation. The transport of molecules across placental membranes is lengthily listed (Miller, Koszalka and Brent) and some studies of cell surface antigens in mammalian developments are reviewed by Edidin.

The development in only three organ systems is covered, and in each a somewhat pedestrian note is struck. Hot or controversial issues have not been stirred up in these reviews about the heart (Manasek), limb bud (Ede), or immune system (Goldschneider and Barton).

All in all, the editors and reviewers have done a fair job. The publishers, however, can be severely criticised. The book costs ninety dollars, has many typing errors and is inadequately indexed. I have recommended this book to our library, but they cannot afford it.

Joan Feldman

Joan Feldman is a Research Fellow in Zoology at University College, London, UK.

of astronomy"; "fissile radioactive materials could become concentrated by geological processes into a natural atomic pile, which could occasionally blow up", and a host of other non sequiturs. Many facts pop out at us like rabbits from a hat accompanied by a similar aura of surprise and lack of explanation. The book also uses jargon freely. The reader, having no glossary, has to turn to other works for definitions of such terms as Hohmann transfers, coriolis force, grey-sphere radiative equilibrium, body tides, bond albedo and so on. Also in evidence is Firsoff's dislike of professional scientists. For example, "the idea of an inert Moon is still widely held among scientific officialdom, often in the teeth of overwhelming evidence against it". In discussing a dark pattern seen on one of the rocks on Venus pictured by Venera 9 Firsoff puts forward the idea that this could be a lichen growth-a genus from a high temperature biology based on sulphuric acid as the solvent : "of course this is speculation" Firsoff writes, "and speculation is a dirty word in professional circles--but many of the hypotheses that have been given an official accolade are even less well founded".

This book gives an entirely wrong impression of present knowledge of the Solar System and of the state of mind of the scientific enquirer who is presently investigating the planets. Not only do 1 advise people not to buy this book, I will go further, don't even bother to open it.

David W. Hughes

David $W$. Hughes is Lecturer in Astronomy and Physics in the Department of Physics at the University of Sheffield, UK.

\section{Pursuit rotor performance}

Remininiscence, Motivation and Personality. A Case study in Experimental Psychology. By H. J. Eysenck and C. D. Frith. Pp. xxi +430 . (Plenum: New York and London; 1977.) \$33.

Thrs self-proclaimed "case study in experimental psychology" is concerned exclusively with the phenomenon of reminiscence as observed in studies of pursuit rotor performance. The pursuit rotor is a piece of apparatus which requires a human subject to keep a stylus in contact with a small target on a rotating turntable. The reminiscence effect refers to a gain in performanceand possibily in learning-when a rest period is introduced after a period of practice. The authors' introduction asserts that "whether the accumulated work done by generations of students on the problems discussed in this book has been worth the trouble must be left to the reader to judge." The authors are presumably optimistic about the reader's verdict; but not, I think, with good reason.

The major virtue of the pursuit rotor is said to be the replicability of the data which it generates- - "perhaps the most reliable set of observations in experimental psychology." The pursuit rotor, we are told, is "the psycnologists' favorite piece of apparatus." These claims seem absurdly parochial, but even if taken seriously they are offset by the authors' remark-in criticising a study which disagrees with their views-that "the literature on reminiscence is especially rich in 'failures to replicate." "

The book does, however, provide a detailed history of research on this narrow problem. The same studies which the authors had earlier interpreted in terms of "inhibition" theory are now interpreted in terms of "consolidation" theory. The fit of the new theory to data, like that of the old, is not so striking as to compel belief. The theoretical concepts used have not arisen from the study of reminiscence in the pursuit rotor task, but have been imported from general psychology. There is no shortage of available 'explanatory' concepts.

This work will be of interest, and possibly of value, to those who are concerned with the pursuit rotor and related matters. There are, however, many experimental psychologists who would have preferred a more fertile subject for such extensive case study.

Leon J. Kamin

Leon J. Kamin is Professor of Psychology at Princeton University, New Jersey. 